\title{
Ergebnisse von Qualitätskontrollen der individuellen Patientendosen in der Radioonkologie*
}

\author{
Jakob Roth ${ }^{1}$
}

\begin{abstract}
Hintergrund: Neben den Bestrahlungsgeräten und Planungssystemen müssen auch die individuellen Patientendosen einer Qualitätssicherung unterzogen werden. Die Sicherstellung der Dosisapplikation besteht aus einem Zweistufenkonzept: der Kontrolle der geplanten Dosis und der In-vivo-Dosimetrie.

Methodik: Im Gegensatz zur Bestrahlungsplanung, wo die Parameter für eine gewünschte Dosisverteilung und Dosis im Zielvolumen bestimmt werden, wird bei der Kontrolle die Dosis im ICRU-Punkt aus den individuellen Einstellparametern berechnet. Dies erfolgt mit einem einfachen Excel-Programm. Zusätzlich wird bei jeder Erstbestrahlung die Eintrittsdosis auf der Oberfläche des Patienten für jedes einzelne Bestrahlungsfeld mit einer Halbleiterdiode gemessen und mit dem Sollwert aus der Bestrahlungsplanung verglichen.

Ergebnisse: Bei einer vorgegebenen Toleranz von 4\% für die Übereinstimmung der Dosen im ICRU-Punkt lag der Anteil der Bestrahlungspläne mit einer größeren Abweichung bei 2,1\% für Photonenbestrahlungen. Die Toleranz zwischen der gemessenen Eintrittsdosis und der in der Planung berechneten Dosis lag bei $\pm 5 \%$ für Photonenstrahlung ohne Keilfilter und bei $\pm 10 \%$ für Photonenstrahlung mit Keilfilterfeldern sowie für Elektronenstrahlung. Bei 14\% aller Bestrahlungsfelder wurden die vorgegebenen Toleranzen überschritten, ohne Berücksichtigung allfälliger Wiederholungsmessungen: 19\% bei Photonenfeldern ohne Keilfilter, $6 \%$ mit Keilfilter, $9 \%$ bei Elektronenfeldern.

Schlussfolgerung: Da Übertragungs- und Einstellfehler nicht ausgeschlossen werden können, sind patientenbezogene Kontrollen erforderlich. Sie sollen einfach, aussagekräftig und eindeutig sein. Ihre personelle und methodische Unabhängigkeit muss vorausgesetzt werden. Bei Überschreitung der Toleranz müssen Maßnahmen ergriffen werden. Dies muss bei der Festlegung von Toleranzen berücksichtigt werden.
\end{abstract}

Schlüsselwörter: Qualitätssicherung $\cdot$ Hochvolttherapie $\cdot$ Dosiskontrolle $\cdot$ Bestrahlungsplanung $\cdot$ In-vivo-Dosimetrie

Strahlenther Onkol 2008;184:505-9

DOI 10.1007/s00066-008-1866-7

\section{Results of Quality Controls for Individual Doses to Patients in Radiation Oncology}

Background: Along with the treatment equipment and the planning system the individual doses to the patient also have to be part of quality assurance. To make sure that the dose application to the patient is correct, a two-step concept is used: the check of the planned dose and the in vivo dosimetry.

Methods: In contrast to the treatment planning where the necessary parameters for a desirable dose distribution and dose are determined in the target volume, the dose in the ICRU point is calculated from the individual setup parameters. This is done by using an Excel Macro program. Additionally, on the occasion of the first treatment session, the entrance dose on the patient surface is measured for each separate treatment field with a semiconductor diode. This dose is compared to the reference value from the treatment planning.

Results: Given a tolerance level of $4 \%$ for the correspondence of the doses in the ICRU point, $2.1 \%$ of the photon treatment plans showed a bigger deviation. The tolerance of the deviation of the measured entrance dose from the dose calculated in the treatment planning was set to $\pm 5 \%$ for photons without wedge and to $\pm 10 \%$ for photons with wedge or for electrons. $14 \%$ of all treatment fields exceeded the given tolerances without taking possible repeated measurements into account. The corresponding figures for photon fields without wedge, with wedge and for electron fields were $19 \%, 6 \%$ and $9 \%$, respectively.

Conclusion: Patient-related checks are essential since transcription and setup errors cannot be excluded. The checks have to be simple, meaningful and unambiguous. One must assume their independence of the individual and the method. If the tolerances are exceeded, actions have to be taken. This has to be taken into account when the tolerances are established.

Key Words: Quality assurance · High-voltage radiotherapy · Dose control · Dose checks · Treatment planning · In vivo dosimetry

\footnotetext{
*Der Inhalt dieser Publikation wurde als Vortrag auf der Dreiländertagung Medizinphysik 2007 in Bern, Schweiz, präsentiert.
}

${ }^{1}$ Radiologische Physik, Radio-Onkologie, Universitätsspital Basel, Schweiz.

Eingang: 14. Februar 2008; Annahme: 9. Juli 2008 


\section{Einleitung}

Die Qualitätssicherung bei den Bestrahlungen beschränkt sich nicht nur auf die einzelnen Geräte, sondern berücksichtigt auch die patientenbezogene Bestrahlungsplanung und die applizierten Dosen am Patienten. Diese sollen in das Qualitätssicherungssystem miteinbezogen werden. Wichtige Voraussetzungen für eine sinnvolle Kontrolle der Bestrahlungsplanung sind personelle und methodische Unabhängigkeiten sowie eine individuelle Überprüfung der geplanten Dosen [13]. Verschiedene Publikationen weisen auf die Notwendigkeit von kritischen Überprüfungen der Ergebnisse hin, insbesondere bei neuen Bestrahlungsplanungssystemen und für komplexe Bestrahlungen [1, 7, 8, 10, 14, 17]. Bestrahlungstechniken wie intensitätsmodulierte Strahlentherapie(IMRT), Stereotaxie und Ganzkörperbestrahlungen werden in dieser Arbeit nicht berücksichtigt.

Für die Sicherstellung der vorbestimmten Dosisapplikation am Patienten hat sich ein Zweistufenkonzept bewährt [11]. Der erste Teil besteht in der von der Bestrahlungsplanung unabhängigen Kontrolle der Dosis im ICRU-Punkt und deren Vergleich mit der geplanten Dosis [12]. Im zweiten Teil wird mit einer In-vivo-Messung die Eintrittsdosis bei jedem Bestrahlungsfeld mindestens bei der Erstbestrahlung und nach einer Planänderung durch die MTRA bestimmt und mit der zuvor berechneten Dosis verglichen [9]. Mit diesem Konzept sollten zumindest grobe Fehler ausgeschlossen werden. Damit kann auch zur Sicherheit der Arbeit des Personals beigetragen werden.

Die Motivation für die patientenbezogenen Qualitätskontrollen liegt in der rechtzeitigen Entdeckung von Fehlern, der Bestätigung einer im Allgemeinen guten Arbeit sowie der Bewusstseinsbildung für eine realistische Genauigkeit bei der Dosisapplikation (unter Berücksichtigung der Messungenauigkeit). Das Zweistufenkonzept für die individuelle Dosiskontrolle wird in der Radio-Onkologie Basel, Schweiz, seit 1992 angewendet und wurde mehrmals verbessert bzw. angepasst.

\section{Methodik}

\section{Kontrolle der Dosis im ICRU-Punkt}

Während es das Ziel der Bestrahlungsplanung ist, die Parameter zu bestimmen, um eine gewünschte Dosisverteilung und Dosis im Zielvolumen zu realisieren, wird bei der Kontrolle der Dosis im ICRU-Punkt der umgekehrte Weg gewählt. Dabei wird von den individuellen Einstellparametern am Linearbeschleuniger ausgegangen und daraus mit Hilfe eines einfachen PC-Programms die Dosis im ICRU-Punkt bestimmt. Die geplante und die bei der Kontrolle be- rechnete Dosis sollen innerhalb von $4 \%$ übereinstimmen, damit der Bestrahlungsplan freigegeben wird.

Alle neuen und geänderten Bestrahlungspläne werden täglich durch einen Medizinphysiker kontrolliert. Wenn nur eine Fraktion oder wenige Fraktionen mit hohen Dosen erfolgen, so ist eine vorgängige Kontrolle besonders wichtig. Anderenfalls soll sie innerhalb von maximal drei Bestrahlungsfraktionen erfolgen. Für die Überprüfung eines Plans benötigt man im Durchschnitt etwa 10 min, ist aber stark abhängig von der Anzahl der Felder und der Komplexität des Plans. Die Verantwortung für das Planungszielvolumen sowie für die entsprechende Dosis im Tumor und im gesunden Gewebe liegt bei der Bestrahlungsplanung bzw. beim zuständigen Radioonkologen. Der Medizinphysiker übernimmt mit der Kontrolle die Verantwortung dafür, dass mit den dokumentierten Bestrahlungsparametern die geplante Dosis pro Fraktion im ICRU-Punkt innerhalb einer vorgegebenen Toleranz von $\pm 4 \%$ realisiert werden kann. Ist die Abweichung größer, so muss die Ursache dafür gesucht werden. Der festgelegte Toleranzwert ergab sich aus der Diskussion zwischen Radioonkologen und Medizinphysikern und wird als realistische Größe beurteilt. Er wurde im Laufe der Zeit von 5\% auf 4\% reduziert.

Die Berechnung der Dosis im ICRU-Punkt unter Berücksichtigung der individuellen Bestrahlungsparameter wird mit einem selbst erstellten Excel-Programm durchgeführt [12]. Dessen Bildschirmdarstellung ist in Abbildung 1 wiedergegeben. Für jede Strahlenqualität an einem Bestrahlungsgerät sind dafür die folgenden Daten gespeichert: Tiefendosentabellen für die FOD (Fokus-Oberflächen-Distanz) von 100 $\mathrm{cm}$ bei verschiedenen Feldgrößen, Dosis-Monitor-Verhältnisse bei verschiedenen Feldgrößen für FOD $=100 \mathrm{~cm}$ im

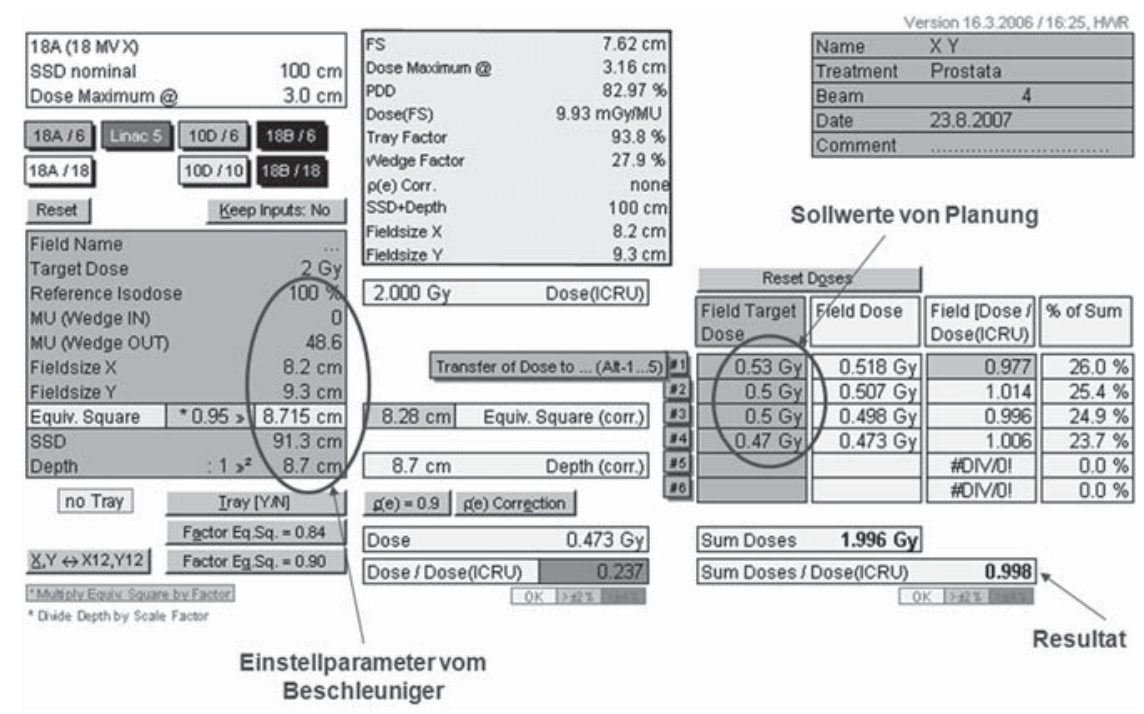

Abbildung 1. Bildschirmdarstellung beim Excel-Programm „MU Dose“ für die Dosiskontrolle in der Hochvolttherapie.

Figure 1. Screen shot of Excel Macro program "MU Dose" for checking the dose in high-voltage therapy. 
Dosismaximum, Schwächungsfaktoren für Keilfilter und Plexiglas-Trägerplatte. Die folgenden individuellen Einstellparameter von jedem Bestrahlungsfeld werden bei der Kontrolle berücksichtigt: Strahlenqualität, FOD, Feldgröße (im Isozentrum) unter Berücksichtigung einer irregulären Form, Keilfilter, Plexiglas-Trägerplatte, Anzahl der Monitoreinheiten und allenfalls durchstrahlte Inhomogenitäten im Körper. Die Dosis im ICRU-Punkt wird mit Hilfe des quadratischen Abstandsgesetzes und durch Interpolationen aus den gespeicherten Datensätzen berechnet.

Bei der Kontrolle der Dosis im ICRU-Punkt wird von vereinfachten Annahmen ausgegangen. So erfolgen die Berechnungen generell in der Feldmitte für ein Wasserphantom bei senkrecht auftreffendem Zentralstrahl. Inhomogenitäten werden nur berücksichtigt, sofern eine Luftkavität (Dichte $0 \mathrm{~g} / \mathrm{cm}^{3}$ ), Lunge (Dichte $0,35 \mathrm{~g} / \mathrm{cm}^{3}$ ) oder Mammagewebe (Dichte $0,9 \mathrm{~g} / \mathrm{cm}^{3}$ ) durchstrahlt wird. Bei einer irregulären Feldform wird mit Hilfe von Musterfeldformen ein Korrekturfaktor für die äquivalente Feldgröße geschätzt, welcher zwischen 0,7 (bei großer bzw. stark irregulärer Ausblockung) und 1,0 (nur geringe oder keine Ausblockung) liegt. Der Faktor ist relativ unkritisch, insbesondere bei Feldgrößen ab $10 \mathrm{~cm} \times 10 \mathrm{~cm}$.

\section{In-vivo-Dosimetrie}

Für die Methode der In-vivo-Dosimetrie wird auf die Literatur verwiesen [1, 4, 5, 7, 15, 16]. Die Messung in der Feldmitte auf der Oberfläche des Patienten bei jedem einzelnen Bestrahlungsfeld erfolgt mit einer Halbleiterdiode (System DPD-3, Fa.

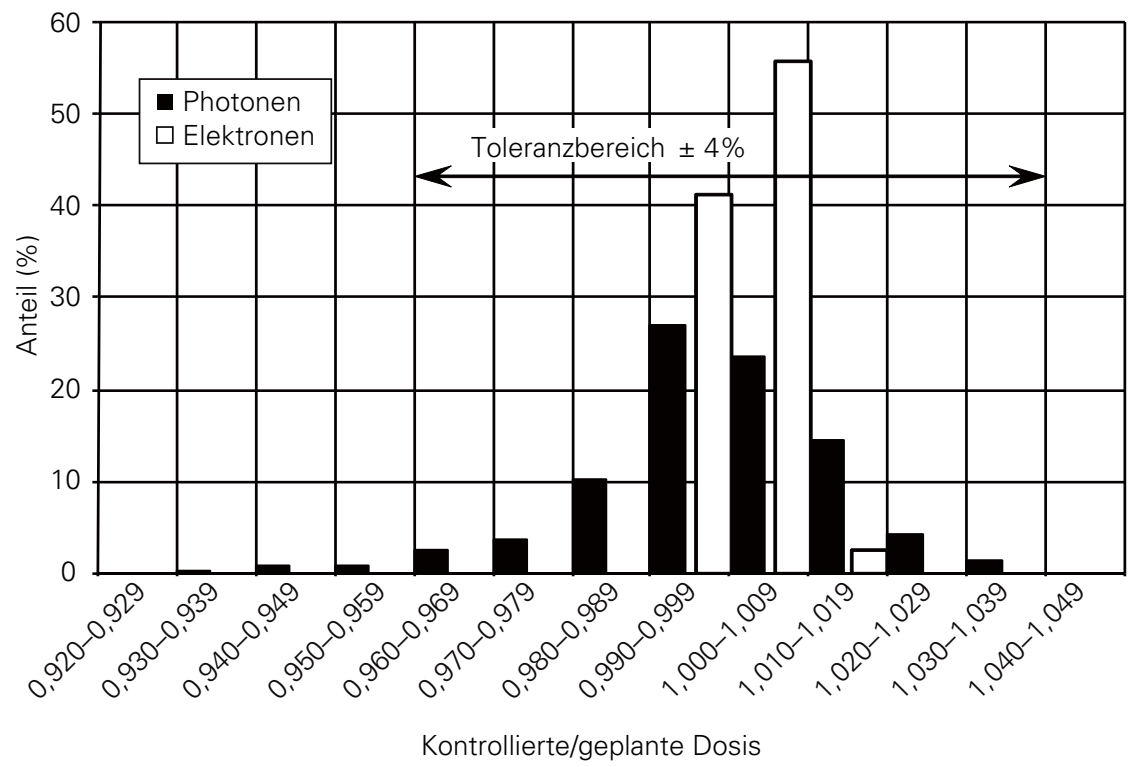

Abbildung 2. Ergebnisse der Dosiskontrollen in der Hochvolttherapie. Dargestellt ist die Häufigkeit versus Differenz zwischen kontrollierten und in der Bestrahlungsplanung bestimmten Dosen.

Figure 2. Results of the dose checks in high-voltage therapy. The frequency of occurrence versus the difference between the measured entrance doses and the calculated entrance doses from treatment planning is shown.
Scanditronix/Wellhöfer, Schwarzenbruck), welche sich für Photonen- und Elektronenstrahlung unterscheidet. Die Detektoren enthalten Aufbaumaterial und werden monatlich mit Hilfe einer Ionisationskammer so kalibriert, dass der Anzeigewert der Dosis in der Tiefe des Maximums entspricht, abhängig von der Strahlenqualität. Die Genauigkeit der Kalibrierung liegt bei 3\%. Die Toleranz zwischen Ist- (gemessen) und Solldosis (berechnet) für ein Bestrahlungsfeld in der Tiefe des Dosismaximums wurde mit $\pm 5 \%$ festgelegt, wobei folgende Ausnahmen bestehen: Die Toleranz beträgt $\pm 10 \%$ bei Keilfilterfeldern (wegen der kritischen Positionierung der Diode und des steilen Dosisgradienten) und bei Elektronenstrahlung. Die Sollwerte für die einzelnen Bestrahlungsfelder im Dosismaximum werden während der Bestrahlungsplanung bestimmt.

Bei Photonenstrahlung wird die Messung einmal wiederholt, wenn die Abweichung bei der ersten Messung zwischen $5 \%$ und $10 \%$ bzw. bei einem Keilfilterfeld zwischen $10 \%$ und $15 \%$ liegt. Bei Elektronenstrahlung soll die Messung grundsätzlich nur einmal durchgeführt werden, weil die Diode eine Schattenwirkung im darunter liegenden Gewebe verursacht, vor allem bei Energien bis $10 \mathrm{MeV}$. Ist die Abweichung größer als die vorgegebene Toleranz, so muss der Grund für die Differenz geklärt werden.

\section{Ergebnisse}

\section{Kontrolle der Dosis im ICRU-Punkt}

Die vorgegebene Toleranz zwischen kontrollierter und geplanter Dosis liegt bei $\pm 4 \%$. Die Auswertung der Ergebnisse von Dosiskontrollen bei perkutanen Bestrahlungen erfolgte über einen Zeitraum von 6 Monaten (ca. 1200 Bestrahlungspläne). Die Ergebnisse der Auswertung sind in Abbildung 2 dargestellt. Für Photonenbestrahlungen lag der Anteil der Pläne mit einer Abweichung von $>4 \%$ bei $2,1 \%$. Das mittlere Verhältnis von kontrollierter zu geplanter Dosis lag bei 0,999 für Photonenund bei 1,000 für Elektronenbestrahlungen.

Die hauptsächlichen Gründe für Dosisabweichungen außerhalb der Toleranz von $>4 \%$ bestanden in

- Übertragungsfehlern (zwischen der Planung und dem Verifikationssystem des Beschleunigers),

- falschen oder ungenauen Angaben der FOD,

- unnötiger bzw. vergessener Berücksichtigung von Trägerplatten.

Der Anteil beträgt je etwa 25\%. Vereinzelt wurden auch Dokumentations- oder Zuordnungsfehler bzw. Verwechslungen von Feldern festgestellt. 
In-vivo-Dosimetrie

Die Auswertung der Resultate von etwa 500 Messungen bei Erstbestrahlungen ist in Abbildung 3 dargestellt. Es wurde festgestellt, dass bei $14 \%$ aller Bestrahlungsfelder die vorgegebenen Toleranzen überschritten wurden: $19 \%$ bei Photonenfeldern ohne Keilfilter, $6 \%$ mit Keilfilter, $9 \%$ bei Elektronenfeldern. Dabei sind die Resultate von allfälligen Wiederholungsmessungen nicht berücksichtigt. Das Verhältnis von Ist- zu Sollwert lag im Durchschnitt bei 0,996 für Photonen- und 0,983 für Elektronenbestrahlungen.

Unter anderen wurden folgende Gründe für Abweichungen außerhalb der vorgegebenen Toleranzen festgestellt:

- falsche FOD,

- nicht oder irrtümlich berücksichtigte Trägerplatte,

- falsche Strahlenenergie,

- falsche Anzahl an Monitoreinheiten,

- Verwechslung von Feldern.

In zahlreichen Fällen können die Abweichungen jedoch messtechnisch bedingt sein, oder die Bestimmung des Sollwerts in der Bestrahlungsplanung erfolgte ungenau bzw. nicht am Messort.

Die Abweichungen außerhalb der festgelegten Toleranzen betrafen zu einem großen Teil Bestrahlungen im Kopf-Hals-Bereich, wo die genaue bzw. reproduzierbare Positionierung der Messsonde oft schwierig ist. Auch eine Maske mit Lufteinschlüssen kann die gemessene Dosis beeinflussen. Zudem ergeben komplexe Bestrahlungstechniken häufigere Abweichungen außerhalb der Toleranzwerte.

\section{Diskussion}

Kontrolle der Dosis im ICRU-Punkt

Das im Wesentlichen seit 1993 eingesetzte PC-Programm erlaubt eine einfache und benutzerfreundliche Anwendung. Die individuelle Kontrolle wird unabhängig von der Bestrahlungsplanung durchgeführt und benutzt einen anderen, einfacheren Rechenalgorithmus sowie eigens gemessene Ausgangsdaten. Obwohl zahlreiche Vereinfachungen zugrunde gelegt und die wenigen gespeicherten Maschinendaten für den gesamten Bereich der verwendeten Parameter umgerechnet werden, lässt sich feststellen, dass auch bei komplexen Bestrahlungsplänen die Dosen in den meisten Fällen innerhalb von $4 \%$ übereinstimmen. Es ist erstaunlich, dass der Vergleich der von einem einfachen Dosisberechnungsprogramm und von einem komplexen Bestrahlungsplanungssystem berechneten Dosen grundsätzlich innerhalb der vorgegebenen Toleranz liegt. Ist die Abweichung größer, so kann von einem Fehler ausgegangen werden.
Ein Grund für systematische Abweichungen liegt darin, dass bei den Kontrollen die Dosen im Allgemeinen in Wasser berechnet werden. Der Unterschied zu den in Gewebe mit einer Dichte von $0,95 \mathrm{~g} / \mathrm{cm}^{3}$ berechneten Dosen beträgt je nach Energie zwischen 1\% und 2\%.

Der ermittelte Anteil von 2,1\% für Dosisabweichungen $>4 \%$ kann einerseits als klein beurteilt werden. Andererseits wird damit aber auch gezeigt, dass eine Kontrolle notwendig ist. Bei einer früheren Auswertung der Kontrollergebnisse von Bestrahlungsplänen im Jahre 1999 [12] lag der Anteil der Dosisabweichungen bei etwa $2,5 \%$, allerdings für eine Toleranz von 5\%. Somit wurde eine Verbesserung erzielt.

\section{In-vivo-Dosimetrie}

Als Grund für die Abweichungen fand sich oft eine ungenaue Bestimmung der Sollwerte in der Bestrahlungsplanung. Eine Abweichung kann aber auch messtechnisch bedingt sein oder bei der Sollwertbestimmung liegen. So ist die Empfindlichkeit des In-vivo-Detektors nach der Kalibrierung unter Standardbedingungen von der FOD und von der äquivalenten Feldgröße abhängig [2,9]. Die Abweichung kann dadurch bis zu etwa $10 \%$ betragen.

Die Überschreitung der vorgegebenen Toleranzen in $19 \%$ aller Bestrahlungen zeigt auch hier die Notwendigkeit der Kontrolle. Allerdings muss beachtet werden, dass es sich dabei um Erstmessungen handelt, welche anlässlich der Erstbestrahlungen erfolgen. In den meisten Fällen liegt die Wiederholungsmessung innerhalb des Toleranzwerts. Ein Grund für die zahlreichen Überschreitungen liegt in der festgelegten 
Toleranzgrenze für die Dosisabweichung von 5\% bei Photonenbestrahlungen ohne Keilfilter. Würde sie ebenfalls auf $10 \%$ erhöht, so resultiert eine Abweichung nur in 3\%, insgesamt bei allen Bestrahlungen in 4\%. Eine frühere Auswertung im Jahre 1994 ergab bei 11\% der Photonenfelder und 23\% der Elektronenfelder zu große Abweichungen [9]. Die Zunahme der Abweichungen bei Photonenstrahlung ist vermutlich auf die komplexeren Bestrahlungstechniken zurückzuführen.

\section{Schlussfolgerung}

Die Qualitätskontrollen am Bestrahlungsplanungssystem und an den Bestrahlungsgeräten sind eine Voraussetzung für eine korrekte Dosisapplikation am Patienten. Zusätzlich sind aber auch patientenbezogene Kontrollen notwendig, da Übertragungs- und Einstellfehler nicht ausgeschlossen werden können. Die Kontrollen sollen einfach, aber aussagekräftig und eindeutig sein. Sie müssen unabhängig von der Person, der Methode (z.B. Algorithmus) und den zugrunde gelegten Daten erfolgen. Für die IMRT sind die beschriebenen Kontrollen nur beschränkt anwendbar. Die Überprüfung der in der Bestrahlungsplanung bestimmten Dosis erfolgt üblicherweise durch Phantommessungen $[1,6,16]$.

Bei Überschreitung der Toleranz müssen Maßnahmen ergriffen werden. Deshalb müssen die Toleranzen realistisch gewählt werden. Da Fehler bei den hohen Dosen große Auswirkungen haben können, müssen mit der Qualitätssicherung die Schwachstellen erfasst und aufgezeigt werden. Insbesondere systematische Fehler müssen eruiert werden. Daraus können entsprechende Konsequenzen gezogen werden. Deshalb sollten die Ergebnisse wie auch die Methoden einer Qualitätssicherung regelmäßig einer kritischen Beurteilung unterzogen werden. Nicht berücksichtigt werden mit den beschriebenen Kontrollen Positionierungs- und Lokalisierungsungenauigkeiten [3].

Es ist wichtig, dass eine periodische Rückmeldung über die Kontrollergebnisse an das Personal erfolgt. Die Kontrollen mit einem guten Ergebnis können dem Personal eine korrekte und sorgfältige Arbeit attestieren. Aus solchen mit einem unbefriedigenden Ergebnis sollten entsprechende Lehren gezogen werden. Fehler sind menschlich und immer möglich, insbesondere bei komplexen Systemen, bei Stress, bei der Übertragung von Daten usw. Sie müssen aber gefunden und korrigiert werden.

\section{Literatur}

1. AAPM Report No 87. Diode in vivo dosimetry for patients receiving external beam radiation therapy. Radiation Therapy Committee Task Group No 62. Madison: Medical Physics Publishing, 2005.
2. Blyth CM, Macleod AS, Thwaites DJ. A pilot study of the use of in vivo dosimetry for quality assurance in radiotherapy. Radiography 1997;3:131-42.

3. El-Bassiouni M, Davis JB, El-Atta I, et al. Target motion variability and on-line positioning accuration during external-beam radiation therapy of prostate carcinoma with an endorectal balloon device. Strahlenther Onkol 2006;182:531-6.

4. Essers $M$, Mijnheer $B J$. In vivo dosimetry during external photon beam radiotherapy. Int J Radiat Oncol Biol Phys 1999;43:245-59.

5. Frye DMD, Rustgi SN. Diode verification of routine electron-beam treatments. Med Dosim 1999;24:43-8.

6. Georg D, Kroupa B, Georg P, et al. Inverse planning - a comparative intersystem and interpatient constraint study. Strahlenther Onkol 2006:182:473-80.

7. Huyskens DP, Bogaerts R, Verstraete J, et al. Practical guidelines for the implementation of in vivo dosimetry with diodes in external radiotherapy with photon beams (entrance dose). ESTRO, Physics for Clinical Radiotherapy Booklet No 5. Leuven: Garant, 2001.

8. Mika S, Christ G. Experimentelle Validierung eines Monte-Carlo-basierten Bestrahlungsplanungssystems für Elektronenstrahlung. Strahlenther Onkol 2007:183:150-6.

9. Nemec HW, Roth J, Roser HW, et al. In-vivo-Dosimetrie bei Erstbestrahlungen: Ergebnisse und Probleme. In: Leemann B, Seiler R, Thum P, Hrsg. Tagungsbericht SGSMP 1998. Luzern: SGSMP, 1999:207-11.

10. Nijsten SM, Mijnheer BJ, Dekker AL, et al. Routine individualised patient dosimetry using electronic portal imaging devices. Radiother Oncol 2007; 82:65-75.

11. Roth J. Ergebnisse der Qualitätskontrollen von individuellen Patientendosen in der Radio-Onkologie. In: Fix MK, Manser P, Mini R, Hrsg. Medizinphysik 2007. Bern: Dreiländertagung, Eigenverlag (ISBN 3-908-125-42-1), 2007:32-3.

12. Roth J, Roser HW. Zur Kontrolle der individuellen Bestrahlungsplanung: Stimmt die Dosis im ICRU-Punkt? In: Gfirtner H, Hrsg. Medizinische Physik 1999. Passau: DGMP, 1999:113-4.

13. Roth J, Roser HW. Von den physikalischen Kontrollen zum Qualitätssicherungskonzept der Radio-Onkologie Basel. In: Kneschaurek P, Hrsg. Medizinische Physik 2000. München: DGMP, 2000:117-8.

14. Scherf C, Scherer J, Bogner L. Verifikation und Anwendung des voxelbasierten Monte-Carlo-(VMC++-)Elektronen-Dosismoduls von Oncentra Master Plan. Strahlenther Onkol 2007;183:81-8.

15. Shakeshaft JT, Morgan HM, Simpson PD. In vivo dosimetry using diodes as a quality control tool: experience of 2 years and 2000 patients. Br J Radiol 1999;72:891-5.

16. Swinnen A, Verstraete J, Huyskens D. Feasibility study of entrance in vivo dose measurements with mailed thermoluminescence detectors. Radiother Oncol 2004;73:89-96.

17. Voordeckers M, Gossens H, Rutten J, et al. The implementation of in vivo dosimetry in a small radiotherapy department. Radiother Oncol 1998;47:45-8.

\author{
Korrespondenanschrift \\ Prof. Dr. Jakob Roth \\ Kirchackerstraße 28 \\ 4422 Arisdorf \\ Schweiz \\ Telefon (+41/61) 811-2541, Fax -813-9407 \\ E-Mail:jakob.roth@bluewin.ch
}

